\title{
CCL21 modulates the migration of NSCL cancer by changing the concentration of intracellular $\mathrm{Ca}^{2+}$
}

\author{
JUN LIU, LEI ZHANG and CHANGLI WANG \\ Lung Cancer Center, Tianjin Medical University Cancer Institute and Hospital, Tianjin 300060, P.R. China
}

Received September 23, 2011; Accepted October 31, 2011

DOI: $10.3892 /$ or.2011.1528

\begin{abstract}
Recurrence and metastasis are the major factors associated with the poor prognosis of non-small cell lung cancer (NSCLC). It has been shown that multiple chemokines and their receptors are related to the progression and metastasis of NSCLC. The aim of this study was to conduct an investigation into whether CCL21 and its receptor, CCR7, play a role in NSCLC invasion and metastasis. We used Western blotting, immunocytochemistry and flow cytometry to detect CCR7 protein expression in four NSCLC cell lines EKVX, HOP-62, NCI-H23 and Slu-01; and we conducted a cell migration experiment to observe the pseudopodia formation and mobility of the lung cancer cells. The concentration of intracellular calcium was measured by fluorescence microscopy. CCR7 protein was positively expressed in the four NSCLC cell lines EKVX, HOP-62, NCI-H23 and Slu-01. Following CCL21 stimulation, obvious pseudopodia formation of lung cancer cells was observed. The cell migration experiment showed that following incubation with CCL21, the number of EKVX cells which passed through the polycarbonate micro-porous filter membranes also increased to an obvious extent. After CCL21 incubation, the intracellular $\mathrm{Ca}^{2+}$ level of the EKVX cells increased to an obvious extent. Chemokine CCL21 facilitates the migration of lung cancer by changing the concentration of intracellular $\mathrm{Ca}^{2+}$. The CCL21-CCR7 axis may play an important role in NSCLC invasion and metastasis. It may also be a potential target for NSCLC therapy or for prevention of the recurrence and metastasis of NSCLC.
\end{abstract}

\section{Introduction}

About 8-9 out of 10 cases of all lung cancers are non-small cell lung cancer (NSCLC) being the most common cancer in men and the third most common cancer in women worldwide,

Correspondence to: Dr Changli Wang, Lung Cancer Center, Tianjin Medical University Cancer Institute and Hospital, Tianjin 300060, P.R. China

E-mail: changliwangtj@163.com

Key words: CCL21 chemokine receptor, non-small cell lung cancer, intracellular $\mathrm{Ca}^{2+}$ with more than 1.1 million new cases of NSCLC reported annually and nearly 1.2 million deaths each year (1-3) and the incidence continues to increase (4). After clinical diagnosis of lung cancer, only about $20 \%$ patients benefit from curative surgical therapies such as lung resection. Furthermore, a recurrence rate as high as around $65 \%$ is seen within five years and the survival rate is only $30-40 \%$ at five years post-operatively. NSCLC recurrence and metastasis are the main causes of treatment failure and the high fatality rate of this cancer. So the major factors associated with the poor prognosis of NSCLC are the high frequency of tumor recurrence and metastasis after curative resection (5-8). Therefore, a deeper understanding of the molecular mechanism of lung cancer occurrence and metastasis is critical to finding new treatment methods and to achieving longer survival rates in NSCLC patients.

The chemokines are a super-family of chemo-attractant cytokines, which are grouped into four families $(\mathrm{C}, \mathrm{CC}$, $\mathrm{CXC}$ and $\mathrm{CX} 3 \mathrm{C}$ ) based on the pattern of cysteine residues in the ligands. Their receptors belong to a family of specific $G$ protein-coupled seven-transmembrane domain receptors (9-13). It has been demonstrated that chemokines and their receptors play a key role in the recruitment of leukocytes to the site of inflammation. Recent studies have shown that they are also correlated to tumor progression, invasion and metastasis of many other kinds of tumors such as breast cancer (14-18), gastric cancer, colorectal cancer, pancreatic cancer, lung cancer, oral squamous cell carcinoma, nasopharyngeal carcinoma, prostate cancer and neuroectodermal tumors (19-23).

It has been demonstrated that the chemokine, CCL21, and its receptor, CCR7, are highly expressed in human lung cancer tissues (24-26). Furthermore, Liu et al (27) demonstrated the major contribution of the CCR7-CCL21 axis to non-small cell lung cancer progression. Olkhanud et al (28) used the artificial micro-RNA (miRNA) technique to study the effects of CCR7 expression on NSCLC cell invasiveness and growth in vitro and found that CCR7 gene expression knockdown inhibited the invasiveness of human NSCLC cells. However, the precise mechanism of the CCL21-CCR7 axis that enables lung cancer cell migration and invasion is not clear and needs further investigation.

In the present study, we have confirmed the enhanced surface expression of CCR7 on NSCLC cell lines and investigated whether the CCL21-CCR7 axis is involved in the migration and invasion of NSCLC cell lines in vitro. The 
change of intracellular $\mathrm{Ca}^{2+}$ levels may play an important role in the function of the CCL21-CCR7 axis.

\section{Materials and methods}

Reagents and cell culture. Reagents and their respective sources used in this study are as follows: RPMI-1640 medium, fetal bovine serum were obtained from Sigma; rabbit antihuman CCR1, CCR7, CXCR4, CXCR3 monoclonal antibody, goat anti-mouse IgG, DAB color kit (Dako ) and CCL21 were from Invitrogen.

Four human NSCLC cell lines, including EKVX, HOP-62, NCI-H23 and Slu-01 were obtained from the China Centre for Type Culture Collection (CCTCC). These cell lines were maintained in RPMI-1640 medium, supplemented with $10 \%$ heat-inactivated fetal bovine serum in a humidified incubator at $37^{\circ} \mathrm{C}$ in $5 \% \mathrm{CO}_{2}$.

Western blot analysis. Cells were incubated with lysis buffer [50 mM N-(2-hydroxyethyl)-piperazine-N9-(2-ethanesulfonic acid), $150 \mathrm{mM} \mathrm{NaCl}, 1.5 \mathrm{mM} \mathrm{MgCl}_{2}, 0.5 \mathrm{mM}$ ethylenediaminetetraacetic acid, $10 \%$ glycerol, $1 \%$ Triton X-100 (Sigma), $10 \mathrm{mM}$ NaF, $1 \mathrm{mM}$ dithiothreitol and $1 \mathrm{mM}$ phenylmethylsulfonyl fluoride] for $15 \mathrm{~min}$ and then were alternately frozen $\left(-80^{\circ} \mathrm{C}\right)$ and thawed three times to rupture the cell membranes. Subsequently, the lysates was centrifuged at $12000 \mathrm{~g}$ for $5 \mathrm{~min}$ to pellet the cell membranes or subcellular organelles. Protein concentrations were determined by a standard Bradford assay (Bio-Rad). Equal amounts of protein $(20 \mathrm{mg})$ from each cell line were subjected to Western blot analysis. The antibodies used for probing were mouse anti-CCR1, CCR7, CXCR4, CXCR3 [1:1000; sc-1517 (Santa Cruz Biotechnology, Inc.)] and mouse anti-actin [1:5000; 51K4888 (Sigma)].

Immunocytochemistry (ICC). Four cultured human NSCLC cell lines were seeded into the eight wells of a Lab-Tec chamber slide and washed with PBS three times; then $4 \%$ paraformaldehyde was added and fixed for $10 \mathrm{~min}$, then washed with PBS several more times. Normal serum closed fluid was added, and then it was stored at room temperature for $30 \mathrm{~min}$. The slides were incubated sequentially with primary rabbit anti-human CCR7 antibody (1:100 dilution) at $4^{\circ} \mathrm{C}$ overnight and with goat anti-rabbit IgG for $45 \mathrm{~min}$ at $37^{\circ} \mathrm{C}$. PBS washed three times, each time for $5 \mathrm{~min}$. A DAB test kit was used for color reaction, after which it was washed with distilled water to eliminate any color. As a control, the first antibody was replaced with normal rabbit IgG.

Flow cytometry. Cultured NSCLC cells were harvested and the first step was incubation in a washing buffer containing $5 \%$ normal goat serum in order to prevent any non-specific binding. For antibody staining, $5 \times 10^{5}$ cells were incubated with either $20 \mu \mathrm{g} / \mathrm{ml}$ of rabbit anti-human CCR7 or the same concentration of normal rabbit IgG as a control. After being washed twice with a washing buffer, the cells were incubated with FITC-labeled goat anti-rabbit IgG $(5 \mu \mathrm{g} / \mathrm{ml})$. The cells were then filtered thorough nylon mesh after being washed twice. All incubations were done for $20 \mathrm{~min}$ on ice followed by two washings in a washing buffer. A total of $1 \times 10^{4}$-gated events was collected from each flow cytometry sample.

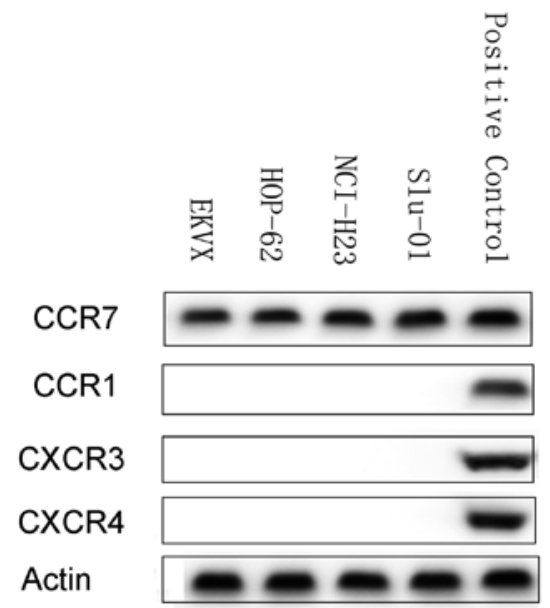

Figure 1. Western blot analysis detected chemokine receptor CCR7, CXCR4, CXCR3 and CCR1 protein expression in four NSCLC cell lines EKVX, HOP-62, NCI-H23 and Slu-01. $\beta$-actin served as a control for sample loading. Representative results from three independent experiments are shown.

Pseudopodia observation and migration assay. We observed the pseudopodia formation of EKVX cells incubated with CCL21 by microscopy. EKVX cells were preseeded and incubated with $900 \mathrm{nmol} \mathrm{CCL} 21$ for $2 \mathrm{~h}$ and they were then examined by microscopy.

Tumor cell migration was assayed in 24-well-culture chambers using $8 \mu \mathrm{m}$ pore polycarbonate microporous filter membrane (Sigma) inserts pre-coated with Matrigel and 1\% fetal bovine serum. The upper chamber was seeded with $100 \mu \mathrm{l}\left(1.5 \times 10^{5}\right.$ cells/well $)$ in the experimental group, and the CCR7 ligand-CCL21 (50, 150, 250, 350 or $450 \mathrm{ng} / \mathrm{ml})$ were added to the chamber. After incubation at $37^{\circ} \mathrm{C}$ for $18 \mathrm{~h}$, the migrated cells attracted by chemokines were attached beneath the membrane. After the filters were removed, the upper surface was scraped, fixed with methanol and stained with hematoxylin. The number of cells was counted as the number of migrated cells per eight fields at x200 magnification.

Intracellular calcium assay. HOP-62 cells were incubated with CCL21 (900 nm) and PBS (control) in the eight wells of a Lab-Tec chamber slide, then washed with D-Hanks before the addition of the fluorescent probe Fluo-3/AM (final concentration $5 \mu \mathrm{mol} / \mathrm{l}$ ), dark incubated at $37^{\circ} \mathrm{C}$ for $40 \mathrm{~min}$. After the Fluo-3/AM hydrolysis into Fluo-3 in the cytoplasm, the concentration of intracellular calcium was measured and quantified using a microscope.

Statistical methods. All data were expressed as the means \pm SD and were analyzed statistically using the Student's t-test, with p-values $<0.05$ considered statistically significant. All statistical analyses were performed using SPSS 13.0 software.

\section{Results}

Chemokine receptor protein expression in NSCLC cell lines. We first examined the protein expression of chemokine receptors in several NSCLC cell lines with Western blotting. Among the chemokine receptor protein that we examined, CCR7 protein was detected in all NSCLC cell lines (Fig. 1). 
(1)

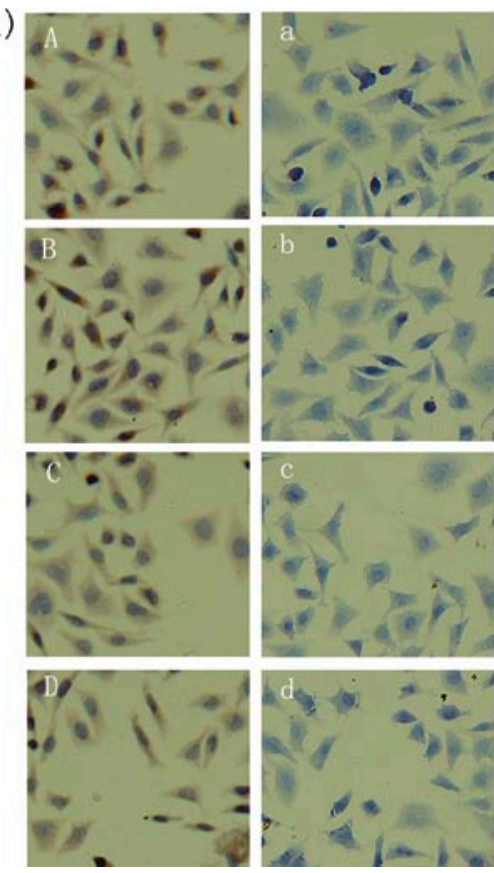

(2)

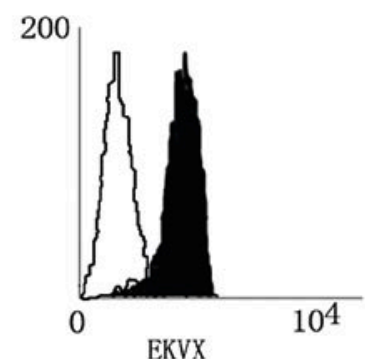

200

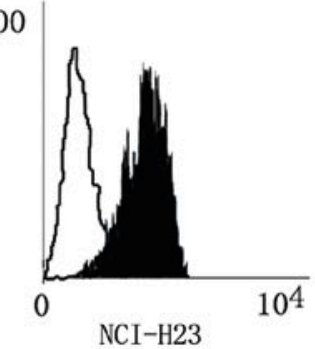

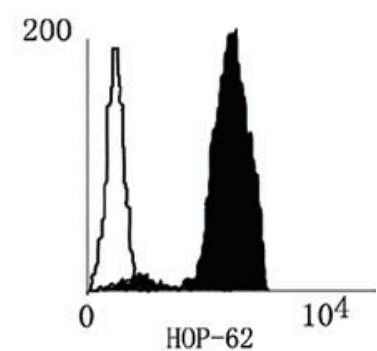

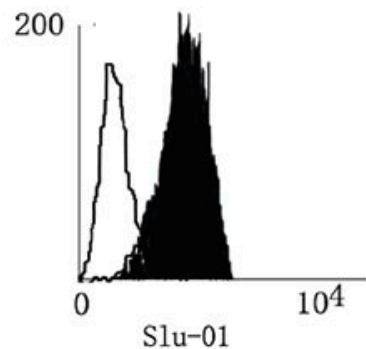

Figure 2. (1) CCR7 protein expression of NSCLC cell lines by immunohistochemistry. Positive expression of CCR7 in EKVX, HOP-62, NCI-H23 and Slu-01 were determined by immunocytochemistry using specific polyclonal rabbit anti-human CCR7 antibodies (A-D). As a control, the first antibody was replaced with normal rabbit IgG (a-d). (2) Flow cytometric analysis of cell-surface CCR7 expression by NSCLC cell lines. Cells (5 to 10x10 $)$ were incubated with rabbit anti-human CCR7 or normal rabbit IgG as a control. Representative results from three independent experiments are shown.

Immunohistochemical staining of CCR7 in NSCLC cell lines. Immunohistochemical staining was used to establish whether NSCLC cell lines expressed CCR7 protein. We detected positive immune reactions in all the cell lines that we examined [Fig. 2(1A-D)]. The use of control antibodies did not give rise to positive reactions at all [Fig. 2(1a-d)]. These results implied that the observed immunoreactivities were specific. Furthermore, flow cytometric analysis also detected CCR7 expression on all NSCLC cell lines [Fig. 2(2)], indicating that CCR7 was expressed on the cell surface of these NSCLC cell lines.

Chemokine-mediated morphological change and migration of EKVX cells. Reorganization of morphological change was an major event in the migratory response to chemokine. In the absence of CCL21, EKVX cells appeared rounded (Fig. 3A). However, in the presence of CCL21, pseudopodia formation was observed in EKVX cells (Fig. 3B), Transwell migration assays were performed to investigate the mobilizing effect of CCL21 on EKVX cells in vitro. We found that CCL21 caused directional migration of EKVX cells in a dose-dependent manner. MCF-7 cells, which express very low levels of CCR7, did not migrate in response to any concentration of CCL21 (Fig. 3C).

Intracellular $\mathrm{Ca}^{2+}$ levels. After the Fluo-3/AM hydrolysis into Fluo-3 in the cytoplasm, the concentration of intracellular calcium was measured using a fluorescence microscope. In relation to the fluorescence intensity of intracellular $\mathrm{Ca}^{2+}$ concentration, the fluorescence was more intensive in the CCL21 incubated-group than that in control group $\left({ }^{*} \mathrm{p}<0.05\right)$ (Fig. 4).

\section{Discussion}

Tumor metastasis is a multi-step process involving many pathways, including the release of tumor cells from the primary cancer which then enter into vascular or lymphatic circulation, and extravasate from circulation to a distant site (29-32). Furthermore, the metastasis of many kinds of tumors have organ specificity. It has been demonstrated that the migration of tumor cells is directed by multiple chemokines and their receptors, and this procedure is similar to the leukocyte trafficking mediated by chemokine (33-36).

It has been confirmed that multiple chemokines and their receptors correlate with the progression and metastasis of NSCLC. Significant overexpression of CCL20 in the tumor tissues and marked overexpression of the corresponding receptor CCR6 in the tumor invasion front of NSCLC patients compared to a normal liver have been reported $(15,18,36)$. It has been demonstrated that IL-8, a member of the superfamily of CXC chemokines, is related to metastatic potential, such as vessel invasion of NSCLC $(25,26,28,29,34,38)$. It has also been demonstrated that CCL21, and its receptor, CCR7, are highly expressed in human NSCLC tissue. Furthermore, using the model of CCL21- or CCR7-deficient mice treated with $\mathrm{N}$-nitrosodiethylamine (DEN), demonstrates that the CCR7-CCL21 axis plays an important role in lung cancer (30). Whether the CCL21-CCR7 axis plays a role in NSCLC invasion and metastasis is not clear.

In the present study, we found that CCR7 protein was positively expressed in the four NSCLC cell lines EKVX, HOP-62, NCI-H23 and Slu-01. After stimulation with CCL21, obvious pseudopodia formation of NSCLC cells was observed with a fluorescence microscope. A cell chemotasis and migra- 

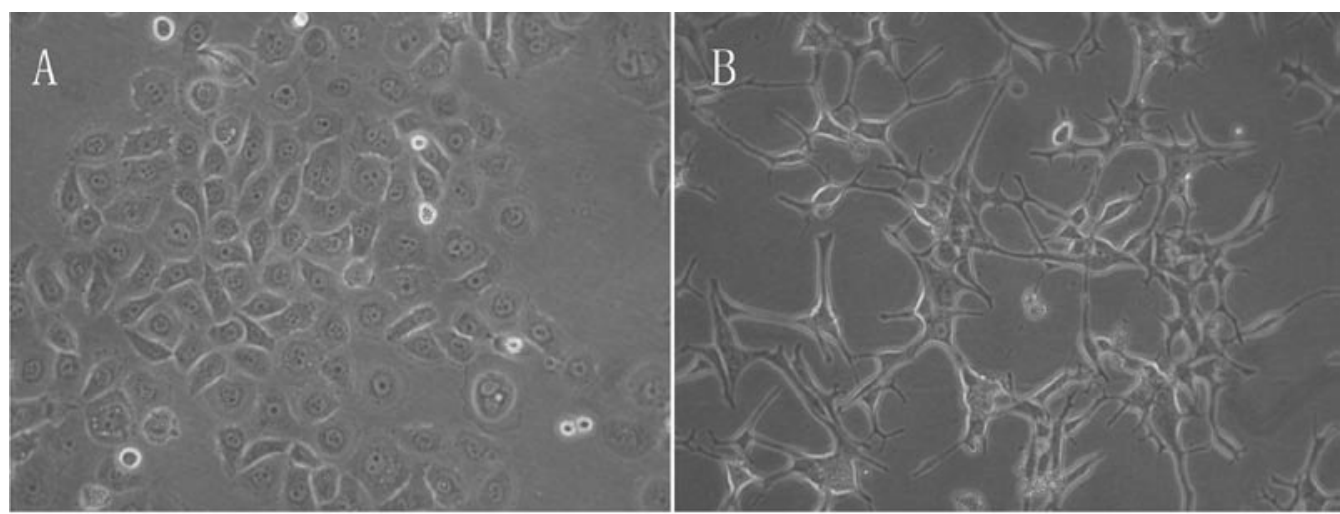

C
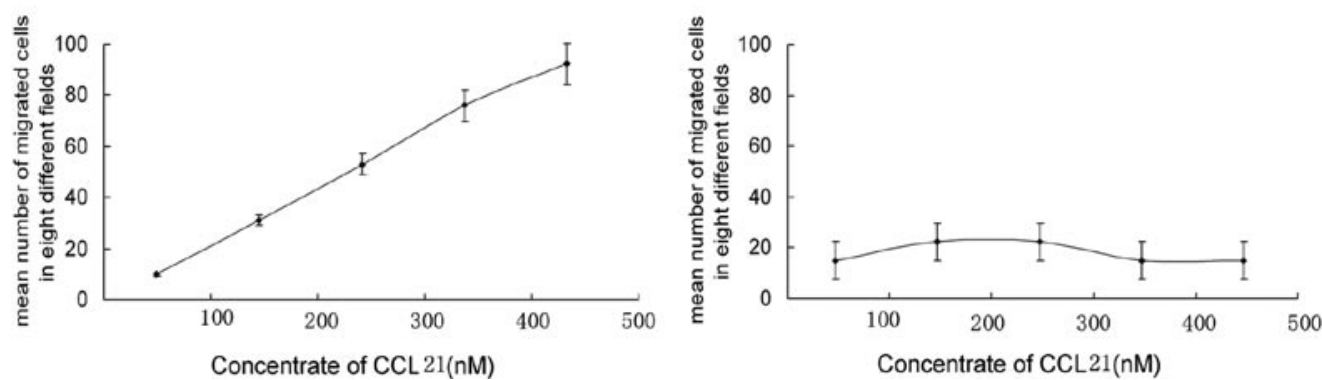

Figure 3. Microscopy for EKVX cells stimulated with CCL21. EKVX cells were incubated (A) without or (B) with $900 \mathrm{nmol} \mathrm{CCL21} \mathrm{for} 2 \mathrm{~h}$. After stimulation with CCL21, formation of pseudopodia on EKVX cells was observed. Migration of EKVX cells in response to CCL21 stimulation. Data show the number of migrated cells in eight different fields from a representative experiment performed in triplicate and repeated three times with similar results. As a negative control, MCF-7 cells (breast cancer cell line) were examined with the same method (C). All data are presented as the mean \pm SD.
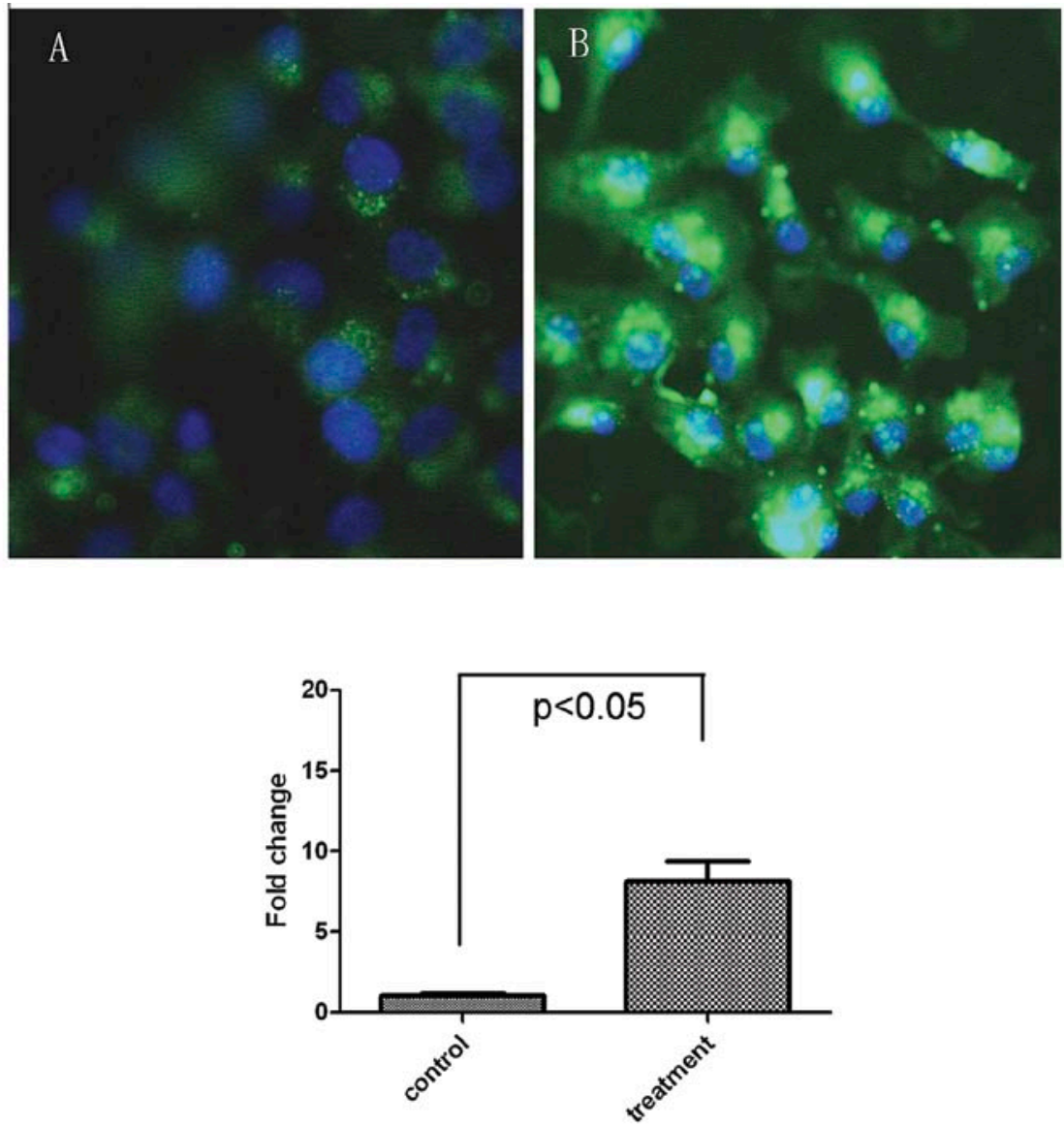

Figure 4. EKVX cells were incubated with CCL21 $(900 \mathrm{~nm})$ and PBS (control) in the eight wells of a Lab-Tec chamber slide. After the Fluo-3/AM hydrolysis into Fluo-3 in the cytoplasm, the concentration of intracellular calcium in control group (A) and CCL21-incubated group (B) were measured and quantified using a microscope. The fluorescence intensity was more intense in the CCL21 incubated-group than that in control group (C) ("p<0.05). All data are presented as the mean \pm SD. 
tion experiment showed that after incubation with CCL21, the number of EKVX cells passing through the membrane increased to an obvious extent. Based on the results, we can presume that CCL21-CCR7 axis may accelerate the migration of NSCLC cells.

Chemokine receptors belong to a family of specific $\mathrm{G}$ protein-coupled seven-transmembrane domain receptors. Activation of chemokine receptors commonly leads to a PLC/ IP3-mediated release of $\mathrm{Ca}^{2+}$ from intracellular stores and results in large increases in cytosolic $\mathrm{Ca}^{2+}$ concentrations in various cell types (39). The change of $\mathrm{Ca}^{2+}$ concentrations will trigger downstream signal transduction pathways. For example, activation of CXCR4 induced glioma cell chemotaxis and could trigger an increase of intracellular $\left[\mathrm{Ca}^{2+}\right]_{i}$. Such an activation could result in the increased production of VEGF by the stimulated tumor cells (18); Yoon et al (18) reported that activation of $\mathrm{CXCR} 4$ by binding toxin-sensitive $\mathrm{G}$ protein, could trigger an increase of intracellular $\left[\mathrm{Ca}^{2+}\right]_{i}$ and cause the activation of PyK2, which controls the activity of the $\mathrm{BK}_{\mathrm{Ca}}$ channels, which then leads to activation of cell proliferation. In our study, to further determine whether the CXCR4 receptor expressed in NSCLC cell lines is functional, the effect of CXCL12 on intracellular calcium $\left[\mathrm{Ca}^{2+}\right]_{\mathrm{i}}$ mobilization was assessed. The concentration of intracellular calcium was measured using a laser scanner microscope, and after CXCL12 incubation the intracellular $\mathrm{Ca}^{2+}$ level of EKVX cells increased to an obvious extent. However, EKVX cells are only one of the CXCR4 expressing NSCLC cell lines, therefore, we will establish these findings using other CXCR4 expressing NSCLC cell lines in the future.

In summary, the present study found the positive expression of functional CCR7 in four human NSCLC cell lines and the activation of CCR7 increased the intracellular $\left[\mathrm{Ca}^{2+}\right]_{\mathrm{i}}$ concentration. Our results suggested that the CCL21/CCR7 axis may play an important role in NSCLC invasion and metastasis. It could also be a potential target for NSCLC therapy or prevention of the recurrence and metastasis of NSCLC.

\section{Acknowledgements}

This study was partly funded by the National Natural Science Funds.

\section{References}

1. Pleasance ED, Stephens PJ, O'Meara S, et al: A small-cell lung cancer genome with complex signatures of tobacco exposure. Nature 463: 184-190, 2010.

2. Khodarev NN, Pitroda SP, Beckett MA, et al: MUC1-induced transcriptional programs associated with tumorigenesis predict outcome in breast and lung cancer. Cancer Res 69: 2833-2837, 2009.

3. Kim WY, Jin Q, Oh SH, et al: Elevated epithelial insulin-like growth factor expression is a risk factor for lung cancer development. Cancer Res 69: 7439-7448, 2009.

4. Takizawa H, Kondo K, Toba H, Kenzaki K, Sakiyama S and Tangoku A: Fluorescence diagnosis of lymph node metastasis of lung cancer in a mouse model. Oncol Rep 22: 17-21, 2009.

5. Germain F, Wai ES, Berthelet E, Truong PT and Lesperance M: Brain metastasis is an early manifestation of distant failure in stage III non-small cell lung cancer patients treated with radical chemoradiation therapy. Am J Clin Oncol 31: 561-566, 2008.

6. Liu Y, Li QC, Miao Y, et al: Ablation of p120-catenin enhances invasion and metastasis of human lung cancer cells. Cancer Sci 100: 441-448, 2009.
7. Ma S, Xu Y, Deng Q and Yu X: Treatment of brain metastasis from non-small cell lung cancer with whole brain radiotherapy and Gefitinib in a Chinese population. Lung Cancer 65: 198-203, 2009.

8. White FA, Jung $\mathrm{H}$ and Miller RJ: Chemokines and the pathophysiology of neuropathic pain. Proc Natl Acad Sci USA 104: 20151-20158, 2007.

9. Di Sciascio MB, Vianale G, Verna N, et al: Eosinophil recruiting chemokines are down-regulated in peripheral blood mononuclear cells of allergic patients treated with deflazacort or desloratadine. Int J Immunopathol Pharmacol 20: 745-751, 2007.

10. Berbaum K, Shanmugam K, Stuchbury G, Wiede F, Korner H and Munch G: Induction of novel cytokines and chemokines by advanced glycation endproducts determined with a cytometric bead array. Cytokine 41: 198-203, 2008.

11. Schmelzer C, Lorenz G, Rimbach G and Doring F: Influence of Coenzyme $\mathrm{Q}$ on release of pro-inflammatory chemokines in the human monocytic cell line THP-1. Biofactors 31: 211-217, 2007.

12. Khandaker MH, Mitchell G, Xu L, et al: Metalloproteinases are involved in lipopolysaccharide- and tumor necrosis factor-alphamediated regulation of CXCR 1 and CXCR 2 chemokine receptor expression. Blood 93: 2173-2185, 1999.

13. Zheng K, Li HY, Su XL, et al: Chemokine receptor CXCR7 regulates the invasion, angiogenesis and tumor growth of human hepatocellular carcinoma cells. J Exp Clin Cancer Res 29: 31, 2010.

14. Villablanca EJ, Raccosta L, Zhou D, et al: Tumor-mediated liver $\mathrm{X}$ receptor-alpha activation inhibits $\mathrm{CC}$ chemokine receptor-7 expression on dendritic cells and dampens antitumor responses. Nat Med 16: 98-105, 2010.

15. Cullen R, Germanov E, Shimaoka T and Johnston B: Enhanced tumor metastasis in response to blockade of the chemokine receptor CXCR6 is overcome by NKT cell activation. J Immunol 183: 5807-5815, 2009.

16. Sung B, Jhurani S, Ahn KS, et al: Zerumbone down-regulates chemokine receptor CXCR4 expression leading to inhibition of CXCL12-induced invasion of breast and pancreatic tumor cells. Cancer Res 68: 8938-8944, 2008.

17. Iida N, Nakamoto Y, Baba T, et al: Tumor cell apoptosis induces tumor-specific immunity in a CC chemokine receptor 1- and 5-dependent manner in mice. J Leukoc Biol 84: 1001-1010, 2008.

18. Yoon Y, Liang Z, Zhang X, et al: CXC chemokine receptor-4 antagonist blocks both growth of primary tumor and metastasis of head and neck cancer in xenograft mouse models. Cancer Res 67: 7518-7524, 2007.

19. Alejo A, Ruiz-Arguello MB, Ho Y, Smith VP, Saraiva M and Alcami A: A chemokine-binding domain in the tumor necrosis factor receptor from variola (smallpox) virus. Proc Natl Acad Sci USA 103: 5995-6000, 2006.

20. Engl T, Relja B, Marian D, et al: CXCR4 chemokine receptor mediates prostate tumor cell adhesion through alpha5 and beta3 integrins. Neoplasia 8: 290-301, 2006.

21. Issa A, Le TX, Shoushtari AN, Shields JD and Swartz MA: Vascular endothelial growth factor- $\mathrm{C}$ and $\mathrm{C}-\mathrm{C}$ chemokine receptor 7 in tumor cell-lymphatic cross-talk promote invasive phenotype. Cancer Res 69: 349-357, 2009.

22. Pasero C, Barbarat B, Just-Landi S, et al: A role for HVEM, but not lymphotoxin-beta receptor, in LIGHT-induced tumor cell death and chemokine production. Eur J Immunol 39: 2502-2514, 2009.

23. Peng W, Ye Y, Rabinovich BA, et al: Transduction of tumorspecific T cells with CXCR2 chemokine receptor improves migration to tumor and antitumor immune responses. Clin Cancer Res 16: 5458-5468, 2010.

24. Hamatake M, Aoki T, Futahashi Y, Urano E, Yamamoto N and Komano J: Ligand-independent higher-order multimerization of CXCR4, a G-protein-coupled chemokine receptor involved in targeted metastasis. Cancer Sci 100: 95-102, 2009.

25. Izraely S, Klein A, Sagi-Assif O, et al: Chemokine-chemokine receptor axes in melanoma brain metastasis. Immunol Lett 130: 107-114, 2010.

26. Kitamura T, Fujishita T, Loetscher $\mathrm{P}$, et al: Inactivation of chemokine (C-C motif) receptor 1 (CCR1) suppresses colon cancer liver metastasis by blocking accumulation of immature myeloid cells in a mouse model. Proc Natl Acad Sci USA 107: 13063-13068, 2010.

27. Liu H, Pan Z, Li A, et al: Roles of chemokine receptor 4 (CXCR4) and chemokine ligand 12 (CXCL12) in metastasis of hepatocellular carcinoma cells. Cell Mol Immunol 5: 373-378, 2008. 
28. Olkhanud PB, Baatar D, Bodogai M, et al: Breast cancer lung metastasis requires expression of chemokine receptor CCR4 and regulatory T cells. Cancer Res 69: 5996-6004, 2009.

29. Pradelli E, Karimdjee-Soilihi B, Michiels JF, et al: Antagonism of chemokine receptor CXCR3 inhibits osteosarcoma metastasis to lungs. Int J Cancer 125: 2586-2594, 2009.

30. Valdivia-Silva JE, Franco-Barraza J, Silva AL, et al: Effect of pro-inflammatory cytokine stimulation on human breast cancer: implications of chemokine receptor expression in cancer metastasis. Cancer Lett 283: 176-185, 2009.

31. Wang L, Yang B, Yang Q, Qiao S, Wang Y and Sun Y: Strong expression of chemokine receptor CXCR4 by renal cell carcinoma cells correlates with metastasis. Clin Exp Metastasis 26: 1049-1054, 2009.

32. Zhang Y, Sun R, Liu B, et al: TLR3 activation inhibits nasopharyngeal carcinoma metastasis via down-regulation of chemokine receptor CXCR4. Cancer Biol Ther 8: 1826-1830, 2009.

33. Croci S, Nicoletti G, Landuzzi L, et al: Expression of a functional CCR7 chemokine receptor inhibits the post-intravasation steps of metastasis in malignant murine mammary cancer cells. Oncol Rep 18: 451-456, 2007.

34. Kawada K, Hosogi H, Sonoshita M, et al: Chemokine receptor CXCR3 promotes colon cancer metastasis to lymph nodes. Oncogene 26: 4679-4688, 2007.
35. Pitkin L, Luangdilok S, Corbishley C, et al: Expression of $\mathrm{CC}$ chemokine receptor 7 in tonsillar cancer predicts cervical nodal metastasis, systemic relapse and survival. Br J Cancer 97: 670-677, 2007.

36. Rubie C, Oliveira-Frick V, Rau B, Schilling M and Wagner M: Chemokine receptor CCR6 expression in colorectal liver metastasis. J Clin Oncol 24: 5173-5174; author reply 5174, 2006.

37. Bartolome RA, Ferreiro S, Miquilena-Colina ME, et al: The chemokine receptor CXCR4 and the metalloproteinase MT1-MMP are mutually required during melanoma metastasis to lungs. Am J Pathol 174: 602-612, 2009.

38. Fukunaga S, Maeda K, Noda E, Inoue T, Wada K and Hirakawa K: Association between expression of vascular endothelial growth factor $\mathrm{C}$, chemokine receptor CXCR4 and lymph node metastasis in colorectal cancer. Oncology 71: 204-211, 2006.

39. Van Deventer HW, Wu QP, Bergstralh DT, et al: C-C chemokine receptor 5 on pulmonary fibrocytes facilitates migration and promotes metastasis via matrix metalloproteinase 9. Am J Pathol 173: $253-264,2008$. 